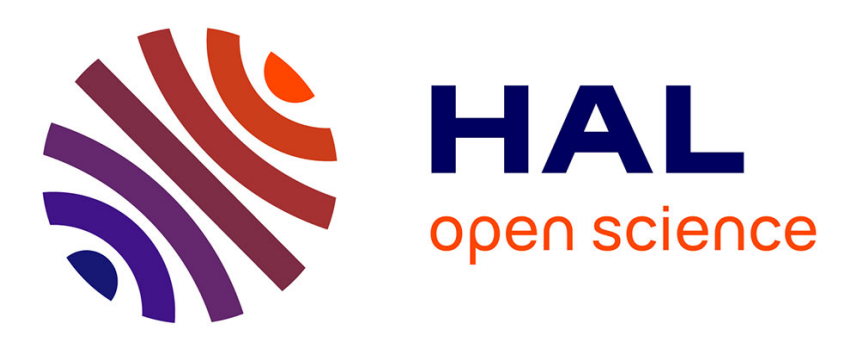

\title{
On the constitutive inequalities and acceleration waves in micropolar media
}

\author{
Holm Altenbach, Victor A. Eremeyev
}

\section{To cite this version:}

Holm Altenbach, Victor A. Eremeyev. On the constitutive inequalities and acceleration waves in micropolar media. PAMM, 2008, 8 (1), pp.10417 - 10418. hal-00824911

\section{HAL Id: hal-00824911 \\ https://hal.science/hal-00824911}

Submitted on 22 May 2013

HAL is a multi-disciplinary open access archive for the deposit and dissemination of scientific research documents, whether they are published or not. The documents may come from teaching and research institutions in France or abroad, or from public or private research centers.
L'archive ouverte pluridisciplinaire HAL, est destinée au dépôt et à la diffusion de documents scientifiques de niveau recherche, publiés ou non, émanant des établissements d'enseignement et de recherche français ou étrangers, des laboratoires publics ou privés. 


\title{
On the constitutive inequalities and acceleration waves in micropolar me- dia
}

\author{
Holm Altenbach ${ }^{1}$ and Victor A. Eremeyev ${ }^{1,2, *}$ \\ ${ }^{1}$ Lehrstuhl für Technische Mechanik, Zentrum für Ingenieurwissenschaften, Martin-Luther-Universität Halle-Wittenberg, \\ D - 06099 Halle (Saale), Germany \\ ${ }^{2}$ South Scientific Center of RASci and South Federal University, 8a, Milchakov St. 8a, 344090 Rostov on Don, Russian \\ Federation
}

\begin{abstract}
Acceleration waves in nonlinear themoelastic micropolar media are considered. We establish the kinematic and dynamic compatibility relations for a singular surface of order 2 in the media. An analogy to the Fresnel-Hadamard-Duhem theorem and an expression for the acoustic tensor are derived. The condition for acceleration wave's propagation is formulated as an algebraic spectral problem. It is shown that the condition coincides with the strong ellipticity of equilibrium equations.
\end{abstract}

\section{Introduction}

The propagation of nonlinear waves in solids is a complex process, analytic solutions for corresponding nonlinear problems are quite rare. However, the problem of propagation of acceleration waves is one of the exceptional cases. An acceleration wave (or wave of weak discontinuity of order 2) is a solution to the equations of motion of the medium that displays discontinuities in the second derivatives on certain surfaces that will be called singular. It means that the acceleration wave is described by the travelling surface which is a carrier of discontinuity jumps of the second derivatives of a solution with respect to space and time whereas the solution and its first derivatives are continuous. From the mathematical point of view, the existence of acceleration waves is closely related to the property of hyperbolicity of the equations of motion (or ellipticity of the equations of statics). The condition of existence of accelerations waves can be reduced to the problem of positivity of all eigenvalues of the algebraic spectral problem for the acoustic tensor for an arbitrary direction of propagation. From the physical point of view, the hyperbolicity of the equations of motion is a natural property of elastic media as well as ellipticity is a natural property of statics. Violation of hyperbolicity (or elipticity) means that discontinuous solutions may appear. Such solutions may model shear-bands, phase transitions, interfaces, fracture, defects, slip surfaces and other phenomena. So, the algebraic criterion for such phenomena is important in the mechanics of materials.

The investigations of acceleration waves in nonlinear elastic and thermoelastic media were performed in many works (see, e.g., [1]). The acceleration waves in elastic micropolar media were considered in [2]. A generalization is presented in [3], where acceleration waves in elastic and viscoelastic micropolar media are studied. The relation between the existence of acceleration waves and the condition of strong ellipticity of the equilibrium equations is established in $[4,5]$.

\section{Acceleration Waves}

The motion of a micropolar media is described by two sets of kinematical variables [6]

$$
x=x(X, t), \quad \chi_{K}=\chi_{K}(X, t), \quad K=1,2,3,
$$

where $x$ is the classical motion, while the vectors $\chi_{K}$ represent the micromotion. Introducing three orthonormal directors $\zeta_{K}$ in the reference configuration by formulas $\zeta_{K}(X) \equiv \chi_{K}(X, 0)$ we further will use the proper orthogonal tensor $H \equiv \chi_{K} \otimes \zeta_{K}$ ( $\operatorname{det} H=1$ ) called microrotation tensor (see, e.g. $[4,7]$ ). $H$ describes microrotation of a particle in the micropolar media.

An acceleration wave (or weak discontinuity wave) is a traveling singular surface $S(t)$ at which the second spatial and time derivatives of the position vector $x$ and of the micro-rotation tensor $H$ have jumps, while $x$ and $H$ together with all their first derivatives are continuous.

We consider two types of acceleration waves. The first one is the homothermal acceleration wave when the temperature field and its first derivatives are continuous at $S(t)$. The second type is the homentropic (or homocaloric) acceleration wave when the entropy field and its first derivatives are continuous at $S(t)$.

For a heat conductive media we have established that the acceleration wave should be homothermal. Homentropic acceleration waves may exist for heat non-conductors. We can use this assumption if we neglect the heat conductivity or consider deformation processes to be very fast.

\footnotetext{
* Corresponding author E-mail: eremeyev@math.rsu.ru.
} 
Using matrix notation, we establish the condition of propagation of homothermal acceleration wave as the spectral problem:

$$
\mathcal{Q}(N) \cdot \boldsymbol{\xi}=V^{2} \mathcal{B} \cdot \boldsymbol{\xi}
$$

where

$$
\boldsymbol{\xi}=\left(a^{\prime}, b^{\prime}\right) \in \mathbb{R}^{6}, a^{\prime}=H^{T} a, b^{\prime}=H^{T} \boldsymbol{b}, \quad \mathcal{Q}(N) \equiv\left[\begin{array}{ll}
\psi, E E\{N\} & \psi, E K\{N\} \\
\psi, K E\{N\} & \psi_{, K K}\{N\}
\end{array}\right], \quad \mathcal{B} \equiv\left[\begin{array}{ll}
\mathbf{1} & 0 \\
\mathbf{0} & \gamma \mathbf{1}
\end{array}\right],
$$

$\psi=\psi(\boldsymbol{E}, \boldsymbol{K}, \theta)$ is the specific free energy, $\theta$ is the temperature, 1 is the unit tensor, $\rho$ is the medium density in the reference configuration, $\rho \gamma$ is the scalar measure of the rotational inertia of a particle, $N$ is the unit normal to $S(t), V$ is the velocity of the wave. $E$ is the Cosserat deformation tensor and $K$ is the wryness tensor that are metric and bending strain measures, respectively. They are defined by the relations $E=H^{T} F, 1 \times \boldsymbol{K}=\left(H^{T} \operatorname{Grad} \boldsymbol{H}\right), \boldsymbol{F}=\operatorname{Grad} x$. Grad is the gradient operator in Lagrangian coordinates.

For arbitrary 4th rank tensor $\boldsymbol{G}$ and vector $\boldsymbol{N}$ that are represented in a Cartesian basis $\boldsymbol{i}_{k}(k=1,2,3)$, we have used the notation $G\{N\} \equiv G_{k l m n} N_{l} N_{n} i_{k} \otimes \boldsymbol{i}_{m} \cdot \mathcal{Q}(N)$ is an analogy to the homothermal acoustic tensor for the micropolar medium. From the existence of the free energy function $\psi$ it follows that $\mathcal{Q}\left(N_{S}\right)$ is symmetric.

Similar to [4,5], we establish the following theorem which is an analogy to the Fresnel-Hadamard-Duhem theorem [1].

Theorem. The condition for the existence of a homothermal acceleration wave for all directions of propagation in a micropolar thermoelastic medium is equivalent to the condition of strong ellipticity of the equilibrium equations of the medium.

Proof. Acceleration waves exist only if all eigenvalues of the spectral problem (2) are positive for any $\boldsymbol{N}$ defining the direction of the wave propagation and it must hold $V^{2}>0$. Properties of positiveness are valid if and only if $\mathcal{Q}$ is positively definite for any values of $N$. So by the definition of positiveness, we have

$$
\xi \cdot \mathcal{Q}(N) \cdot \xi>0, \quad \forall N:|N|=1, \quad \xi \neq 0 .
$$

This is an additional restriction that is imposed on the constitutive relations of the thermoelastic micropolar medium. Inequality (3) coincides with the strong ellipticity condition [4]

$$
\left.\frac{d^{2}}{d \tau^{2}} \psi\left(E+\tau a^{\prime} \otimes N, K+\tau b^{\prime} \otimes N\right)\right|_{\tau=0}>0, \quad \forall \boldsymbol{N}:|N|=1, \quad a^{\prime} \neq \mathbf{0}, \quad b^{\prime} \neq \mathbf{0} .
$$

It completes the proof.

Example. As an example, let us consider a quadratic form as a constitutive equation for the specific free energy. Let us assume the following relation to be valid $\rho \psi=W_{1}(\boldsymbol{E})+W_{2}(\boldsymbol{K})$ with

$$
\begin{aligned}
& 2 W_{1}(E)=\alpha_{1} \operatorname{tr}\left((E-1)(E-1)^{T}\right)+\alpha_{2} \operatorname{tr}\left((E-1)^{2}\right)+\alpha_{3} \operatorname{tr}^{2}(E-1)+\alpha_{0}\left(\theta-\theta_{0}\right) \operatorname{tr}((E-1))+c\left(\theta-\theta_{0}\right)^{2}, \\
& 2 W_{2}(K)=\beta_{1} \operatorname{tr}\left(K K^{T}\right)+\beta_{2} \operatorname{tr}\left(K^{2}\right)+\beta_{3} \operatorname{tr}^{2}(K),
\end{aligned}
$$

where $\alpha_{k}, \beta_{k}(k=1,2,3)$ are elastic constants, $\alpha_{0}$ corresponds to the thermal expansion coefficient, $c$ is the specific heat capacity, and $\theta_{0}$ is the reference temperature. Equation (3) implies the following inequalities

$$
\alpha_{1}>0, \quad \alpha_{1}+\alpha_{2}+\alpha_{3}>0, \quad \beta_{1}>0, \quad \beta_{1}+\beta_{2}+\beta_{3}>0 .
$$

\section{Conclusion}

The conditions for the existence of homothermal acceleration waves in a thermoelastic micropolar medium are established. It is shown that the conditions for the existence of such waves do not depend on the thermoconductivity defined by Fourier's law. Hence the conditions under which the acceleration waves in a thermoelastic micropolar medium with Fourier's law of thermoconductivity propagate coincide with those for wave propagation in the corresponding elastic medium. Thus, we have shown that for analysis of acceleration waves in thermoelastic bodies in a non-homogeneous temperature field it is sufficient to consider only the equations of motion where the temperature is considered as a parameter. The algebraic criterion for the existence of acceleration waves is formulated. This criterion is the strong ellipticity condition. An example on how to check the strong ellipticity condition is presented.

\section{References}

[1] C. Truesdell, Rational Thermodynamics, 2nd ed. (Springer-Verlag, New York et al., 1984).

[2] C.B. Kafadar, and A.C. Eringen, Int. J. Engng. Sci. 9, 271 (1971).

[3] G.A. Maugin, Int. J. Engng. Sci. 12, 143 (1974).

[4] V.A. Eremeyev, Doklady Physics 50, 204 (2005).

[5] V.A. Eremeyev, L.P. Lebedev, and L.A. Rendón, Revista Colombiana de Matemáticas 41, 397 (2007).

[6] A.C. Eringen, Microcontinuum Field Theories. I. Foundations and Solids, (Springer-Verlag, Berlin, Heidelberg, New-York et al., 1999).

[7] H. Altenbach, and P.A. Zhilin, Uspekhi Mekhaniki 11, 107 (1988). 